\title{
Disponibilidade Hídrica Relacionada ao Conteúdo de Nitrogênio e à Produtividade da Alfafa (Medicago sativa L.)
}

\author{
Wladecir Salles de Oliveira1 ${ }^{1}$ Patrícia Perondi Anchão Oliveira², Moacyr Corsi ${ }^{3}$, \\ Paulo César Ocheuze Trivelin ${ }^{4}$, Siu Mui Tsai ${ }^{5}$
}

\begin{abstract}
RESUMO - A variação da abundância isotópica do ${ }^{13} \mathrm{C}$ e fixação biológica do nitrogênio podem ser utilizadas em programas de melhoramento de cultivares de alfafa (Medicago sativa L.), pois contribuem para o entendimento de processos fisiológicos relacionados ao uso da água, fotossíntese, obtenção e uso do nitrogênio, bem como suas interdependências e efeitos sobre a produtividade, eficiência do uso da água e qualidade da biomassa produzida. Nesse sentido, torna-se necessária a avaliação do desempenho sob condições contrastantes em disponibilidade hídrica. Neste estudo, avaliou-se o desempenho de 12 e 6 cultivares de alfafa, respectivamente, em dois experimentos instalados em lisímetros no delineamento estatístico de parcelas subdivididas sob irrigação constante e regime de chuvas. Sob condições controladas, a alfafa responde positivamente à irrigação tanto em relação aos caracteres quantitativos quanto qualitativos e dentre os cultivares estudados, os cultivares Crioula Brasileira e Crioula Chilena apresentaram os melhores desempenhos. Na avaliação de seis meses de duração, não houve correlação entre a discriminação isotópica do carbono (DIC), fixação biológica de nitrogênio (FBN), produção de matéria seca (PMS) e eficiência do uso da água (EUA). Na avaliação de um ano sob irrigação a DIC correlacionou-se negativamente com PMS (-0,92**), EUA (-0,94**) e FBN (-0,79*). A FBN correlacionou-se positivamente com a PMS $\left(0,93^{* *}\right)$ e EUA $\left(0,87^{*}\right)$. Sob regime de chuva não houve correlação entre os parâmetros. Existe uma tendência de correlação positiva entre a PMS e DIC demonstrada em inúmeros trabalhos científicos. Essa tendência pode ser alterada sob limitação de água, limitação ao desenvolvimento radicular ou quando o conjunto de cultivares analisadas é contrastante. Se a correlação permanece de forma positiva invariável, não existe possibilidade de aumento da EUA, uma vez que o aumento da DIC é resultado do aumento da transpiração.
\end{abstract}

Palavras-chave: alfafa, discriminação isotópica de carbono, eficiência do uso da água, fixação biológica de nitrogênio, Sinorhizobium meliloti

\section{Water Availability on Yield and Nitrogen Contents of Alfalfa (Medicago sativa L.)}

ABSTRACT - Variation in the abundance of ${ }^{13} \mathrm{C}$ and biological nitrogen fixation in legume plants such as alfalfa (Medicago sativa L.) can be used in cultivar selection programs allowing a better understanding of the physiological processes related to water use, photosynthesis and biological nitrogen fixation and their interdependence. This would supply data for selection programs for the improvement of dry matter yield (DM), water use efficiency (WUE) and biomass quality. Thus evaluation of the plant under contrasting conditions becomes necessary, therefore in this study, the plants were evaluated under rainfall and irrigated conditions in lysimeters. In this study, 12 and 6 alfalfa cultivars was evaluated respectively in two experiments installed in lysimeters by split-plot statistical design under constant irrigation and regime of rains. The results showed the existence of a complex interdependence between the agronomic characteristics of the alfalfa cultivars. Under controlled conditions the alfalfa responded positively to irrigation, in relation to both quantitative and qualitative characteristics. The cultivars Crioula Chilena and Crioula Brasileira showed the best results. In the first evaluation (after 6 months) correlations between carbon isotopic discrimination (CID), biological nitrogen fixation (BNF), dry matter yield (DM) and water use efficiency (WUE) were not significant. In the second evaluation (after 1 year), under irrigated conditions CID was negatively correlated with DM (-0.92**), WUE $(-0.94 * *)$ and BNF $(-0.79 *)$. BNF was positively correlated with DM $\left(0.93^{* *}\right)$ and WUE $(0.87 *)$. Under non-irrigated conditions correlation between these parameters was not significant. There exists a tendency for a positive correlation between $\mathrm{DM}$ and CID in studies with $\mathrm{C}_{3}$ plants that can be altered when water is limiting, root development is limited or when contrasting cultivars are used. If a positive correlation is maintained, the possibility of increasing the WUE is minimal, since the increase of CID is a result of the increase in transpiration.

Key Words: alfalfa, biological nitrogen fixation, carbon isotopic discrimination, Sinorhizobium meliloti, water use efficiency

\footnotetext{
${ }^{1}$ Aluno de Doutorado, CENA/Universidade de São Paulo. E.mail: wsolivei@carpa.ciagri.usp.br

${ }_{2}^{2}$ Pesquisadora, EMBRAPA/CNPGL - Núcleo Sul. E.mail: ppaolive@cnpgl.embrapa.br

3 Professor Doutor, ESALQ/Universidade de São Paulo. E.mail: moa@carpa.ciagri.usp.br

${ }^{4}$ Professor Doutor, CENA/Universidade de São Paulo. E.mail: pcotrive@cena.usp.br

${ }^{5}$ Professora Doutora, CENA/Universidade de São Paulo. E.mail: tsai@cena.usp.br
} 


\section{Introdução}

A cultura da alfafa é dependente de uma série de fatores como a fertilidade do solo, topografia, práticas culturais e condições climáticas para que seja viável economicamente. O conhecimento aprofundado de manejo de solo, irrigação e beneficiamento da biomassa produzida desestimula o agricultor a plantar alfafa. Na região centro-sul do Brasil, as condições climáticas, exceto a má distribuição das chuvas durante os meses do ano, são propícias à cultura, com índices de produtividade que variam de 15 a 30 Mg.ha.ano-1 de matéria seca (Botrel \& Alvim, 1997; Oliveira et al., 1999).

A utilização de cultivares mais eficientes no uso da água em ambientes com irrigação pode resultar em aumento de produtividade de fitomassa da alfafa. Nesta condição, em que normalmente se atinge a capacidade de campo, a cultura apresenta sua maior intensidade de desenvolvimento na relação: uso da água/produção de biomassa e, as plantas de maior potencial produtivo diferenciam-se economizando ou, muitas vezes, utilizando de forma extravagante a água aplicada. Por outro lado, sob condições de regime de chuvas, alguns cultivares de alta produtividade não apresentam bom desempenho enquanto que outras, pequena redução no ritmo de crescimento em relação à condução com irrigação, demonstrando haver diferença genética entre os cultivares disponíveis para o agricultor.

A eficiência do uso da água (EUA) que refere-se a razão da biomassa produzida e a evapotranspiração $\left(E_{m}\right)$ (Cunha et al., 1994), resume o processo de transpiração da água absorvida pelo sistema radicular e assimilação do carbono via fotossíntese, o que, segundo Farquhar \& Lloyd (1993) pode ser avaliado pela composição dos isótopos de carbono na matéria seca. A avaliação da EUA em lisímetros de balança é laboriosa e sujeita a erros. A avaliação com o auxílio da condutância estomática é pontual, enquanto que a análise da composição isotópica do carbono é o resumo de toda a atividade fotossintética ocorrida no período de crescimento (Johnson \& Tieszen, 1994). A estimativa da demanda de água de uma cultura pode ser obtida pelo coeficiente de cultura de cada fase (Kc) (Doorenbos \& Kassan, 1979). Para a cultura da alfafa a $\mathrm{ET}_{\mathrm{m}}$ encontra-se ao redor de 800 a $1600 \mathrm{~mm} /$ ciclo, que varia de 28 e 45 dias. Os valores de Kc encontram-se em 0,4 após o corte; entre 1,05 e 1,20, no momento do corte; e média de 0,85 a 1,05.
Sob condições de clima mediterrâneo, Metochis (1980) observou variação do Kc da alfafa de 0,75 no inverno e 1,10 no verão de Chipre.

A avaliação indireta da EUA pela discriminação dos isótopos de carbono (DIC) tem sido recomendada por inúmeros autores (Farquhar \& Richards, 1984; Hubick et al., 1986; Condon et al., 1987; Hall et al., 1990; Asseng \& Hsiao, 2000), seja sob condições de chuva ou irrigação artificial. Sob condições de estresse hídrico fatores como capacidade de produção de raízes, capacidade do mesofilo em fixar carbono e condutância estomática, são fundamentais e a relação entre DIC e EUA pode ser afetada (Matus et al., 1995). Plantas com elevados valores de DIC sob irrigação apresentam maior decréscimo da discriminação sob regime de chuva demonstrando a existência de outros fatores relacionados à EUA (White et al., 1990).

A utilização da DIC para estimar a EUA em alfafa vem sendo utilizada como ferramenta em trabalhos de seleção de cultivares (Johnson \& Tieszen, 1994; Ebson et al., 1998). Dentre as vantagens do método, os autores destacam a rapidez da análise isotópica, a integração de performance da planta em uma única análise, a utilização de reduzido volume de material e sua ampla utilização em inúmeros artigos como índice para mensurar a EUA. A razão entre os isótopos estáveis de carbono $\left({ }^{13} \mathrm{C} /{ }^{12} \mathrm{C}\right)$ dentro do sistema solo-planta-atmosfera é resultado de processos físicos, químicos e biológicos que ocorrem nos ciclos biogeoquímicos. Para plantas, a razão isotópica está relacionada à entrada do $\mathrm{CO}_{2}$ nos estômatos, à difusão do $\mathrm{CO}_{2}$ intracelular e às propriedades bioquímicas da enzima RuBisCO, responsável pela fixação do $\mathrm{CO}_{2}$ (Boutton, 1997). Existe variação isotópica entre plantas de diferentes ciclos fotossintéticos, entre plantas de mesmo ciclo fotossitético e até entre plantas de uma mesma espécie. A composição isotópica do carbono é medida em relação a um padrão internacional de concentração conhecida (PDB-Pee Dee Belemnite), conforme descrito por Farquhar et al. (1982) e pode variar de 0 a $-110 \%$. Para estudos com materiais vegetais que possuem como fonte de $\mathrm{CO}_{2}$ a atmosfera, cuja composição isotópica tende a se manter constante em -8\%, Farquhar \& Richards (1984) propuseram a discriminação isotópica (DIC) em relação à razão isotópica do $\mathrm{CO}_{2}$ da atmosfera que é a fonte de $\mathrm{C}$ às plantas para melhor entendimento do fenômeno de empobrecimento isotópico. 
A interação alfafa/Sinorhizobium meliloti, que regula indiretamente a qualidade da fitomassa produzida, é uma das mais efetivas interações entre bactérias fixadoras de nitrogênio dos gêneros (Brady Sino) rhizobium e plantas leguminosas. Admite-se a quantidade média de $450 \mathrm{~kg}$.ha ${ }^{-1}$. ano${ }^{1}$ de nitrogênio como potencial para a obtenção de nitrogênio pela fixação biológica (FBN) (Fishbeck et al., 1987). Entretanto, existem registros de variação entre 120 e $250 \mathrm{~kg} \cdot \mathrm{ha}^{-1}$. ano ${ }^{-1}$ para regiões dos Estados Unidos onde se realizam 2 a 6 cortes por ano (Zhu et al., 1996) e 200 a 650 kg.ha.ano-1 para países como Argentina que realizam até sete cortes anuais (Racca et al., 1998). Nas condições brasileiras a FBN na cultura pode chegar até $900 \mathrm{~kg} \cdot \mathrm{ha}^{-1}$. ano $^{-1}$ (Oliveira et al., 1999).

A avaliação da FBN pela técnica de abundância natural obtida por análise da composição isotópica, apresenta resultados comparáveis a outras como a redução do acetileno ou fertilizantes enriquecidos com ${ }^{15} \mathrm{~N}$ (Shearer \& Kohl, 1997). O aumento da FBN em alfafa depende da identificação de parâmetros relacionados à variação genética que possam ser integrados em programas de melhoramento, como a produção de matéria seca, número e massa de nódulos (Heichel et al., 1984) e atividade da enzima nitrogenase (Jassen et al., 1994) ou, a utilização de metodologias de aplicação direta para quantificação do processo.

O fracionamento isotópico durante o processo de assimilação do nitrogênio é uma interação entre a razão isotópica do nitrogênio absorvido pelo sistema radicular e o nitrogênio obtido via FBN (Mariotti et al., 1980). O efeito isotópico associado à fixação biológica de $\mathrm{N}_{2}$ altera a composição isotópica da biomassa de plantas fixadoras em até $2 \%$ e associada a determinação da razão isotópica de uma planta não fixadora crescida nas mesmas condições, possibilita a quantificação do processo (Shearer \& Khohl, 1997). A seleção de plantas em meio rico de $\mathrm{N}$-mineral resulta em dependência do elemento, pois a alfafa pode utilizar preferencialmente o $\mathrm{N}$ do solo em detrimento do $\mathrm{N}$ obtido via FBN (Teuber \& Phillips, 1986). Na presença de alta concentração de $\mathrm{N}$-mineral, o sistema radicular e a formação de nódulos são afetados prejudicando o processo natural de absorção do nitrogênio, ocasionando um retrocesso nos programas de melhoramento que visam a viabilidade econômica da cultura.
A variação da razão isotópica de $\mathrm{N}\left({ }^{15} \mathrm{~N} /{ }^{14} \mathrm{~N}\right)$, avaliada com o auxílio da determinação da abundância dos isótopos ou via enriquecimento isotópico é ferramenta única de avaliação direta da FBN, principalmente em condições de campo, em que outras técnicas se tornam inviáveis (Hardarson et al., 1984).

O teor de nitrogênio em plantas leguminosas é outra variável importante, por estar relacionado à produção de matéria seca. O processo de fotossíntese é dependente da atividade de enzimas como a Ribulose Bifosfato Carboxilase-Oxigenase (RuBisCO) que constituem grande parcela do $\mathrm{N}$ existente na planta. Para que haja maior atividade fotossintética é necessária a presença de concentração suficiente da enzima RuBisCO (Atkins, 1984).

Esse trabalho foi conduzido com os objetivos de identificar, entre 12 cultivares de alfafa, o mais produtivo, bem como estudar as relações existentes no processo de produção de matéria seca, eficiência do uso da água e fixação biológica de nitrogênio sob as condições climáticas de Piracicaba-SP.

\section{Material e Métodos}

Foram conduzidos dois experimentos no conjunto de lisímetros do Laboratório de Biologia Celular e Molecular do CENA-USP em Piracicaba-SP, onde se avaliaram os efeitos dos fatores: cultivar e regime hídrico (irrigação e regime de chuvas), sobre as variáveis: produção de matéria seca (PMS); eficiência do uso da água (EUA); relação haste/folha (H/F); número de hastes $(\mathrm{NH})$; discriminação dos isótopos de carbono (DIC); teor de nitrogênio (N) e nitrogênio total (NT); teor e nitrogênio total derivado da atmosfera (NDA e NTDA). O conjunto de lisímetros foi composto por doze parcelas separadas por paredes de concreto impermeabilizadas de dimensões de $2 \mathrm{~m}$ de comprimento, 1,5 m de largura e $2 \mathrm{~m}$ de profundidade, sendo $1 \mathrm{~m}$ preenchido com brita $\mathrm{n}^{0} 1$ e $1 \mathrm{~m}$ com solo (Latossolo Vermelho-Amarelo distrófico). As sementes de alfafa foram desinfestadas com álcool $70^{0} \mathrm{GL}$ e hipoclorito de sódio (0,5\%), para a eliminação de microrganismos contaminantes, conforme mencionado por Araújo \& Hungria (1994). Após desinfecção realizou-se a inoculação de Sinorhizobium meliloti SEMIA-116 e peletização com carbonato de cálcio (Somasegaran \& Hoben, 1994). 
Experimento 1 - A acidez e deficiências nutricionais do solo foram corrigidas com base em análise do solo para fins de fertilidade. A calagem empregada, calculada pelo método da saturação por bases, foi de 1,0 Mg/ha-1 de calcário dolomítico PRNT=85, enquanto a adubação foi de $75 \mathrm{~kg} \cdot \mathrm{ha}^{-1}$ de $\mathrm{P}_{2} \mathrm{O}_{5}$ na forma de SFS; $30 \mathrm{~kg}$.ha ${ }^{-1}$ de $\mathrm{K}_{2} \mathrm{O}$ na forma de $\mathrm{KCl}, 30 \mathrm{~kg} \mathrm{ha}^{-1}$ de FTE BR-12 e 90 g.ha $^{-1}$ de molibdênio na forma de molibdato de sódio. Após cada corte foram realizadas adubações de reposição tendo como base o potencial de extração para 2,5 Mg.ha ${ }^{-1}$ de matéria seca a cada corte. Dessa forma, aplicou-se $60 \mathrm{~kg} \cdot \mathrm{ha}^{-1}$ de $\mathrm{P}_{2} \mathrm{O}_{5}$ na forma de SFS, $72 \mathrm{~kg}$.ha ${ }^{-1}$ de $\mathrm{K}_{2} \mathrm{O}$ na forma de $\mathrm{KCl}, 30 \mathrm{~kg}^{-h^{-1}}{ }^{-1}$ fe FTE BR-12 e 50 g.ha ${ }^{-1}$ de molibdênio na forma de molibdato de sódio.

O experimento foi delineado no esquema inteiramente casualizado em parcelas subdivididas, justificada pela necessidade de se isolar o efeito da irrigação. Foram utilizados oito dos doze lisímetros disponíveis. Dentro de cada lisímetro foram semeadas três diferentes cultivares cada qual em quatro linhas de 1,5 m de comprimento, espaçadas em 0,18 m entre si. Dessa maneira, quatro lisímetros foram mantidos sob irrigação e quatro sob regime de chuva. Foram coletadas duas subamostras em cada subparcela. O experimento teve início no mês 06/98. Foram realizados dois cortes de homogeneização após 60 e 90 dias da germinação, quando então teve início o controle de irrigação. A avaliação ocorreu durante o período de 10/98 a 03/99 por meio de seis cortes realizados sempre que as plantas apresentaram $10 \%$ de hastes floridas ou quando os perfilhos basais tivessem de 5 a $8 \mathrm{~cm}$ de altura.

Foram utilizados os cultivares Crioula Chilena, Crioula Brasileira, Victoria, UC-Cíbola, Moapa-69, Mecca-I, Mecca-II, WL-516, WL-605, SW-14, Pioneer-5312, Pioneer-XAI32.

Experimento 2 - Dos 12 cultivares iniciais foram selecionados seis em razão do exposto: Crioula Brasileira é o cultivar de maior difusão entre os produtores brasileiros; Crioula Chilena, maior produção de matéria seca em campo; Pioneer XAI32 apresenta boa produção de matéria seca em campo; Mecca tem bom desempenho quanto a FBN; UC-Cíbola tem boa produção de matéria seca em lisímetros. O experimento foi delineado no esquema inteiramente casualizado em parcelas subdivididas. Dentro de cada um dos doze lisímetros foram semeadas duas diferentes cultivares cada qual em seis linhas de 1,5 m de comprimento, espaçadas em 0,18 m entre si.
Foram coletadas duas subamostras em cada subparcela. O experimento foi avaliado durante o período de aproximadamente um ano, 07/99 a 04/00, com cortes realizados quando as plantas apresentaram 10\% de hastes floridas. Com base na análise química para fins de fertilidade do solo, cada lisímetro recebeu a quantidade de: Lisímetro 5=1,63 Mg.ha ${ }^{-1}$; L6=1,28 Mg.ha' ${ }^{-1}$ : L7= 1,44 Mg.hä ${ }^{-1}$ L8=1,42 Mg.ha-1; L9=1,32 Mg.ha-1; L10=1,63 Mg.ha-1; L11=1,59 Mg.ha-1 e L12=1,06 Mg.ha ${ }^{-1}$, de calcário dolomítico PRNT=98, calculada pelo método da saturação por bases. Aplicou-se ainda $78 \mathrm{~kg}$.ha- ${ }^{-1} \mathrm{de}_{2} \mathrm{O}$ na forma de $\mathrm{KCl}, 30 \mathrm{~kg}^{-h^{-1}}{ }^{-1}$ e FTE BR-12 e 90 g.ha-1 de molibdênio na forma de molibdato de sódio. Após cada corte foram realizadas adubações repositivas iguais as do Experimento 1.

A capacidade de retenção de água do solo foi previamente determinada para uniformização da umidade, indispensável à avaliação das parcelas mantidas sob condições artificiais. A quantidade de água no solo disponível para a planta (AD), foi determinada com o auxílio da fórmula: $\mathrm{AD}=[(\mathrm{CC}-$ PMP). DG] . 300/100, onde: $C \mathrm{C}=29 \%$; $\mathrm{PMP}=17,73 \%$ e $D G=1,22 \mathrm{~g} \cdot \mathrm{cm}^{-3}$. Desse modo, a AD calculada foi igual a 41,25 mm. Portanto, toda vez que se observou 0,5 atm nos tensiômetros aplicou-se por asperssão $1 / 2$ AD ou 20,60 mm.

A altura em cm da coluna de mercúrio correspondente a 0,5 atm indica que cerca da metade da água disponível gasta foi determinada pela equação: $\mathrm{T}=\left(12,6 \mathrm{~h}_{\mathrm{Hg}}-\mathrm{h}_{\mathrm{c}}-\mathrm{H}_{\mathrm{p}}\right)$, em que $\mathrm{h}_{\mathrm{Hg}}=$ altura do mercúrio a partir do nível do reservatório $(\mathrm{cm})$; $\mathrm{h}_{\mathrm{c}}=$ altura do nível de mercúrio em relação ao solo $(\mathrm{cm}) ; \mathrm{h}_{\mathrm{p}}=$ profundidade da cápsula no solo $(\mathrm{cm})$. Foram instalados dois tensiômetros com cápsulas a 10 e $20 \mathrm{~cm}$ de profundidade, nos quais, a altura média de $43 \mathrm{~cm}$ do menísculo em relação à cubeta de mercúrio representou 0,5 atm ou o ponto ideal de início da reposição de água via irrigação.

A cada 28 a 35 dias, o que corresponde a um período necessário para formação, maturação e início do florescimento, as parcelas experimentais foram avaliadas quanto à: produção de matéria seca: realizouse a colheita de quutro subamostras de $0,2 \mathrm{~m}^{2}$ cortadas de 6 a $8 \mathrm{~cm}$ do solo. O material colhido foi seco em estufa com ventilação forçada a $60^{\circ} \mathrm{C}$ durante 72 horas. Antes, as hastes maduras e juvenis foram contadas fornecendo o número de hastes: relação haste/folha: quatro subamostras de cada cultivar foram coletadas para a estimativa da relação haste/folha, as 
quais foram secas em estufa com ventilação forçada a $60^{\circ} \mathrm{C}$ durante 72 horas. Considerou-se haste o caule central e folha, o conjunto de gemas apicais, limbo, pecíolo e flores.

Eficiência do uso da água: obtida pela relação entre a PMS e $\mathrm{ET}_{\mathrm{m}}$, que por sua vez, foi obtida pela relação entre $\mathrm{o} \mathrm{K}_{\mathrm{C}}$ da alfafa e a $\mathrm{ET}_{\mathrm{o}}$ mensurada em tanque tipo Classe $\mathrm{A}$, instalado no posto agrometereológico da ESALQ/USP em Piracicaba-SP.

Teor de nitrogênio: uma subamostra de cada repetição da matéria seca foi moída e homogeneizada para posterior digestão sulfúrica e análise quanto ao teor de nitrogênio por colorimetria.

O nitrogênio total foi obtido pela relação entre o teor de nitrogênio e produção de matéria seca.

Nitrogênio derivado da atmosfera: a porcentagem de nitrogênio na matéria seca oriunda da fixação biológica de nitrogênio foi obtida com a determinação da composição isotópica por espectrometria de massa.

O nitrogênio total derivado da atmosfera foi obtido pela relação entre o nitrogênio derivado da atmosfera e a produção de matéria seca.

Discriminação dos isótopos de carbono: a composição isotópica foi determinada no Laboratório de Isótopos Estáveis do CENA/USP, com o espectrômetro de massa ANCA-IRMS, conforme Barrie \& Prosser (1997). A amostra padrão para controle das análises foi a sacarose de beterraba ( d relativo ao $\mathrm{PDB}=-24.08 \%$ oo $)$. A composição isotópica da atmosfera foi considerada para todos os efeitos como sendo $-8 \%$ oo relação à referência internacional, Pee Dee Belemnite (PDB).

O controle de ervas daninhas foi realizado manualmente, enquanto que o controle de pragas foi realizado em duas ocasiões com o uso do inseticida Deltametrina (0,4 L/ha), para controle de pulgão. Não foi necessário o controle de doenças. A análise estatística dos resultados dos experimentos foi realizada com o software SAS. Avaliou-se a hipótese de igualdade de resposta dos tratamentos pelo teste $\mathrm{F}$ de Snedecor e Fisher e, caso essa hipótese fosse rejeitada, os dados foram submetidos ao teste Tukey de comparações múltiplas de médias, para identificação de possíveis diferenças estatísticas entre as médias obtidas nos tratamentos, conforme descrito por Gomes (1987). As análises de correlação, bem como a determinação dos níveis de significância pelo teste $\mathrm{T}$, foram feitas com o programa SANEST.

\section{Resultados e Discussão}

Nos experimentos, a chuva não foi suficiente para garantir níveis satisfatórios de produção e qualidade proporcionando resultados significativamente inferiores $(\mathrm{P}<0,01)$ ao tratamento irrigado em todas as avaliações (Tabela 1), o que demonstra a dependência da cultura ao fornecimento constante de água.

O desempenho dos cultivares dentro de cada regime hídrico no experimento 1 foi diferenciado. Sob regime de chuva (Tabela 1) os cultivares apresentaram significativo aumento de folhas e teor de nitrogênio e redução do nitrogênio total em razão da menor expansão de hastes e produção de matéria seca. No experimento sob condições de irrigação (Tabela 2), alguns cultivares como a Crioula Chilena, UC-Cíbola, WL-605 e Mecca, apresentaram desempenho significativamente superior em relação às características quantitativas avaliadas $(\mathrm{P}<0,01)$. O mesmo quadro foi observado também, em condições de chuva (Tabela 3), entretanto em menor escala. A correlação dos dados obtidos (Tabela 4) denota estreita relação entre a produção de matéria seca e a eficiência do uso da água $\left(0,98^{* *}\right)$ para os dois regimes hídricos demonstrando similaridade no desempenho sob as condições contrastantes. O excesso de chuvas durante o período (10/98 a 03/99) limitou a necessidade de irrigação, influenciando o desempenho dos cultivares. A produção de matéria seca relacionouse ainda ao N $\left(-0,56^{*}\right)$ e NT $\left(0,94^{* *}\right)$, sob condições de irrigação e a DIC $\left(0,51^{*}\right)$, N\% $\left(-0,68^{* *}\right)$, NT $\left(0,92^{* *}\right)$ e NTDA $\left(0,74^{* *}\right)$, sob chuva. Não houve correlação entre os dados de eficiência do uso da água e discriminação isotópica de carbono, para os dois regimes hídricos, provavelmente, devido ao curto período de avaliação desse experimento.

No experimento 2, o desempenho dos cultivares mantidos sob irrigação, quanto à produção de matéria seca (Tabela 5), foi semelhante, sem diferença significativa. Entretanto, sob regime de chuvas os cultivares Crioula Brasileira e Crioula Chilena apresentaram produtividade significativamente superior $(\mathrm{P}<0,01)$. Conforme verificado por Oliveira 2001, o menor declínio na produtividade durante os meses de inverno para essas cultivares favoreceu a média anual. O mesmo comportamento é verificado para a EUA. Em relação à DIC o cultivar Mecca apresentou os melhores resultados nos dois regimes hídricos, diferenciando-se dos demais. Em relação ao NTDA é válido apontar o efeito negativo do déficit hídrico sobre a FBN do cultivar Pioneer XAI32. Sob condi- 
Tabela 1 - Desempenho de cultivares de alfafa mantidos em lisímetros sob condições de irrigação controlada e regime de chuvas. Média de 4 repetições e 6 cortes no experimento 1 e 4 repetições e 10 cortes no experimento 2

Table 1 - Performance of alfalfa cultivars maintained in lisymeters under rainffed and irrigated conditions. Average of 4 replications and 6 cuts in the experiment 1 and 4 replications and 10 cuts in the experiment 2

\begin{tabular}{|c|c|c|c|c|c|c|c|c|c|}
\hline & & & & & $\begin{array}{l}\text { Variável } \\
\text { Variable }\end{array}$ & & & & \\
\hline & PMS & EUA & $\mathrm{NH}$ & $\mathrm{H} / \mathrm{F}$ & DIC & $\mathrm{N}$ & NT & NDA & NTDA \\
\hline & $\begin{array}{c}D M Y \\
\left({\left.\mathrm{Mg} \cdot \mathrm{ha}^{-1}\right)}^{2}\right.\end{array}$ & $\begin{array}{c}\text { WUE } \\
\left(\mathrm{kg} \cdot \mathrm{mm}^{-1}\right)\end{array}$ & $\begin{array}{c}N S \\
\left(\mathrm{~m}^{2}\right)\end{array}$ & $S / L$ & $\begin{array}{c}C I D \\
\left(\Delta^{13} \mathrm{CPDB}\right)\end{array}$ & $\begin{array}{c}N \\
\text { (\%) }\end{array}$ & $\begin{array}{c}T N \\
\left(\mathrm{~kg}^{-\mathrm{ha}^{-1}}\right)\end{array}$ & $\begin{array}{l}N D A \\
(\%)\end{array}$ & $\begin{array}{c}\text { TNDA } \\
\left(\mathrm{kg} \cdot \mathrm{ha}^{-1}\right)\end{array}$ \\
\hline (Experiment & & & & & & & & & \\
\hline Experiment 1 & & & & & & & & & \\
\hline Irrigado & 2,45 & 2,12 & 643,00 & 0,94 & 22,00 & 3,21 & 77,12 & 85,15 & 67,79 \\
\hline Irrigated & & & & & & & & & \\
\hline Chuva & 1,88 & 1,66 & 559,50 & 0,84 & 21,50 & 3,33 & 59,98 & 69,72 & 42,76 \\
\hline Rainall & & & & & & & & & \\
\hline $\operatorname{DMS}(0,05)$ & 0,80 & 0,07 & 7,14 & 0,02 & 0,08 & 0,05 & 2,47 & 0,40 & 3,50 \\
\hline $\mathrm{CV}$ & 8,05 & 8,72 & 8,66 & 4,80 & 0,78 & 3,37 & 8,86 & 0,84 & 10,63 \\
\hline$\underline{\text { Pr. }}>$ F & $0,0001^{* *}$ & $0,0001^{* *}$ & $=0,0001^{* *}$ & ${ }^{*} 0,0001^{* *}$ & $* \quad 0,0001^{* *}$ & $0,0001^{* *}$ & $0,0001^{* *}$ & $0,0001^{* *}$ & $0,0001^{* *}$ \\
\hline (Experiment & & & & & & & & & \\
\hline Experiment 2 & & & & & & & & & \\
\hline Irrigado & 2,32 & 2,52 & 679 & 0,88 & 21,06 & 3,35 & 73,41 & 82,21 & 60,11 \\
\hline Irrigated & & & & & & & & & \\
\hline Chuva & 1,15 & 1,28 & 542 & 0,62 & 20,56 & 2,98 & 39,40 & 64,13 & 27,74 \\
\hline Rainall & & & & & & & & & \\
\hline DMS $(0,05)$ & 0,14 & 0,15 & 25 & 0,02 & 0,08 & 0,19 & 4,35 & 1,30 & 3,17 \\
\hline $\mathrm{CV}$ & 14,24 & 13,19 & 7,10 & 5,49 & 0,62 & 10,19 & 13,21 & 3,02 & 12,31 \\
\hline Pr. $>$ F & $0,0001^{* *}$ & $0,0001^{* *}$ & $0,0001^{* *}$ & $0,0001^{* *}$ & $* \quad 0,0001 * *$ & $0,0006^{* *}$ & $0,0001 * *$ & $0,0001 * *$ & $0,0001^{* *}$ \\
\hline
\end{tabular}

Tabela 2 - Desempenho de cultivares de alfafa mantidos em lisímetros sob condições de irrigação controlada. Média de 4 repetições e 6 cortes no experimento 1

Table 2 - Performance of alfalfa cultivars maintained in lisymeters under irrigated conditions. Average of 4 replications and 6 cuts in the experiment 1

\begin{tabular}{|c|c|c|c|c|c|c|c|c|c|}
\hline \multirow{3}{*}{$\begin{array}{l}\text { Cultivares } \\
\text { Cultivars }\end{array}$} & \multicolumn{9}{|c|}{$\begin{array}{l}\text { Variável } \\
\text { Variable }\end{array}$} \\
\hline & PMS & EUA & $\mathrm{NH}$ & $\mathrm{H} / \mathrm{F}$ & DIC & $\mathrm{N}$ & NT & NDA & NTDA \\
\hline & $\begin{array}{c}D M Y \\
\left(\mathrm{Mg}^{\left.-\mathrm{ha}^{-1}\right)}\right.\end{array}$ & $\begin{array}{c}W U E \\
\left(\mathrm{~kg} \cdot \mathrm{mm}^{-1}\right)\end{array}$ & $\begin{array}{c}N S \\
\left(\mathrm{~m}^{2}\right)\end{array}$ & $S / L$ & $\begin{array}{c}C I D \\
\left(\Delta^{13} \mathrm{CPDB}\right)\end{array}$ & $\begin{array}{c}N \\
(\%)\end{array}$ & $\begin{array}{c}T N \\
\left(\mathrm{~kg}^{-\mathrm{ha}^{-1}}\right)\end{array}$ & $\begin{array}{c}N D A \\
(\%)\end{array}$ & 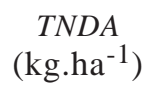 \\
\hline Crioula Brasil & 2,07 & 1,86 & 725 & 0,86 & 22,15 & 3,45 & 71,62 & 85,15 & 59,63 \\
\hline Crioula Chile & 2,68 & 2,26 & 591 & 0,87 & 22,21 & 3,23 & 86,69 & 87,51 & 68,50 \\
\hline Victoria & 2,25 & 1,97 & 715 & 0,83 & 21,91 & 3,48 & 78,29 & 88,66 & 69,84 \\
\hline UC-Cíbola & 2,68 & 2,30 & 658 & 1,06 & 22,02 & 3,18 & 83,96 & 86,44 & 68,44 \\
\hline Моара-69 & 2,44 & 2,10 & 589 & 0,97 & 21,94 & 3,19 & 77,71 & 83,51 & 66,18 \\
\hline WL-516 & 2,37 & 2,04 & 552 & 0,93 & 22,16 & 3,16 & 74,93 & 86,62 & 65,83 \\
\hline WL-605 & 2,76 & 2,42 & 623 & 1,07 & 21,97 & 2,95 & 81,60 & 86,15 & 68,89 \\
\hline SW-14 & 2,60 & 2,20 & 683 & 0,89 & 22,10 & 3,18 & 82,60 & 83,89 & 71,80 \\
\hline Мecca & 2,75 & 2,40 & 647 & 0,95 & 22,22 & 3,17 & 86,73 & 92,24 & 79,42 \\
\hline Месcа II & 2,42 & 2,06 & 634 & 0,90 & 21,92 & 3,20 & 77,38 & 84,36 & 69,82 \\
\hline Pioneer5312 & 2,00 & 1,79 & 670 & 0,92 & 21,64 & 3,25 & 64,89 & 88,75 & 59,72 \\
\hline PioneerXAI32 & 2,39 & 2,12 & 666 & 1,00 & 21,81 & 3,24 & 77,81 & 86,09 & 61,22 \\
\hline DMS(0.05) & 0,42 & 0,37 & 121 & 0,10 & 0,42 & 0,23 & 15,74 & 1,87 & 14,65 \\
\hline $\mathrm{CV}$ & 6,86 & 7,07 & 7,33 & 4,36 & 0,66 & 2,88 & 8,05 & 0,53 & 8,70 \\
\hline Pr. $>$ F & $0,0001^{* *}$ & $0,0001^{* *}$ & $0,0003^{* *}$ & $0,0001 * *$ & $0,0012 * *$ & $0,0001^{* *}$ & $0,0010^{* *}$ & $0,0001^{* *}$ & $0,0013 * *$ \\
\hline
\end{tabular}


Tabela 3 - Desempenho de cultivares de alfafa mantidos em lisímetros sob regime de chuvas. Média de quatro repetições e seis cortes no experimento 1

Table 3 - Performance of alfalfa cultivars maintained in lisymeters under rain-fed conditions. Average of four replications and six cuts in the experiment 1

\begin{tabular}{|c|c|c|c|c|c|c|c|c|c|}
\hline & & & & & $\begin{array}{l}\text { Variável } \\
\text { Variable } \\
\end{array}$ & & & & \\
\hline Cultivares & PMS & EUA & $\mathrm{NH}$ & $\mathrm{H} / \mathrm{F}$ & DIC & $\mathrm{N}$ & NT & NDA & NTDA \\
\hline Cultivars & 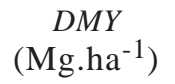 & $\begin{array}{c}W U E \\
\left(\mathrm{~kg} \cdot \mathrm{mm}^{-1}\right)\end{array}$ & $\begin{array}{c}N S \\
\left(\mathrm{~m}^{2}\right)\end{array}$ & $S / L$ & $\begin{array}{c}C I D \\
\left(\Delta^{13} \mathrm{CPDB}\right)\end{array}$ & $\begin{array}{c}N \\
(\%)\end{array}$ & $\begin{array}{c}T N \\
\left(\mathrm{~kg} \cdot \mathrm{ha}^{-1}\right)\end{array}$ & $\begin{array}{l}N D A \\
(\%)\end{array}$ & $\begin{array}{c}\text { TNDA } \\
\left(\mathrm{kg} \cdot \mathrm{ha}^{-1}\right)\end{array}$ \\
\hline Crioula Brasil & 1,88 & 1,70 & 628 & 0,83 & 21,49 & 3,49 & 65,40 & 69,73 & 39,83 \\
\hline Crioula Chile & 1,96 & 1,68 & 584 & 0,77 & 21,68 & 3,33 & 65,10 & 77,93 & 46,32 \\
\hline Victoria & 1,75 & 1,51 & 645 & 0,74 & 21,56 & 3,59 & 62,73 & 79,37 & 49,42 \\
\hline UC-Cíbola & 2,11 & 1,84 & 541 & 0,86 & 21,24 & 3,32 & 69,95 & 79,32 & 45,10 \\
\hline Моapa-69 & 1,52 & 1,31 & 469 & 0,81 & 21,56 & 3,32 & 49,09 & 78,49 & 38,69 \\
\hline WL-516 & 1,74 & 1,57 & 485 & 0,86 & 21,24 & 3,33 & 57,83 & 75,06 & 36,74 \\
\hline WL-605 & 2,18 & 1,94 & 602 & 0,94 & 21,24 & 3,25 & 70,77 & 79,33 & 43,93 \\
\hline SW-14 & 1,99 & 1,76 & 563 & 0,85 & 21,48 & 3,31 & 65,88 & 77,52 & 46,78 \\
\hline Месса & 2,01 & 1,80 & 560 & 0,89 & 21,58 & 3,32 & 67,00 & 68,79 & 41,33 \\
\hline Месcа II & 1,99 & 1,78 & 608 & 0,77 & 21,74 & 3,15 & 62,69 & 78,32 & 39,72 \\
\hline Pioneer 5312 & 1,55 & 1,41 & 580 & 0,91 & 21,42 & 3,60 & 55,87 & 70,83 & 36,84 \\
\hline PioneerXAI32 & 1,86 & 1,63 & 497 & 0,87 & 21,69 & 3,35 & 62,39 & 78,76 & 48,72 \\
\hline DMS (0.05) & 0,41 & 0,37 & 140 & 0,08 & 0,49 & 0,23 & 15,03 & 3,57 & 9,25 \\
\hline $\mathrm{CV}$ & 8,91 & 9,10 & 10,39 & 3,99 & 0,76 & 2,82 & 9,62 & 1,17 & 8,70 \\
\hline Pr. $>$ F & $0,0001^{* *}$ & $0,0001^{* *}$ & $0,0016^{*}$ & $* 0,0001 * *$ & $* \quad 0,0111 *$ & $0,0001^{* *}$ & $0,0008 * *$ & $0,0001^{* *}$ & $0,0001^{* *}$ \\
\hline
\end{tabular}

${ }_{1}$ PMS (DMY) produção de matéria seca (dry matter yield), EUA (WUE) eficiência do uso da água (water use efficiency), NH (NS) número de hastes (number of steams), H/F (S/L) relação haste/folha (steam/leave ratio), DIC (CID) discriminação isotópica de carbono (carbo isotopic discrimination), $\mathrm{N}(\mathrm{N})$ teor de nitrogênio (average of nitrogen), NT (TN) nitrogênio total (total nitrogen), NDA (NDA) nitrogênio derivado da atmosfera (nitrogen derived from atmosphere) e NTDA (TNDA) nitrogênio total derivado da atmosfera (total nitrogen derived from atmosphere) $*(P<0,05){ }^{* *}(P<0,01)$.

Tabela 4 - Correlações lineares entre características de produção e qualidade da alfafa mantidas sob regime de irrigação (acima da diagonal) e regime de chuva (abaixo da diagonal). Média de 4 repetições e 6 cortes no experimento1

Table 4 - Linear correlations among traits associated with yield and quality of the alfalfa maintained under irrigated (above diagonal) and rainfed conditions (below diagonal). Average of 4 replications and 6 cuts in the experiment 1

\begin{tabular}{|c|c|c|c|c|c|c|c|c|c|}
\hline \multirow{3}{*}{$\begin{array}{l}\text { Cultivares } \\
\text { Cultivars }\end{array}$} & \multicolumn{9}{|c|}{$\begin{array}{l}\text { Variável } \\
\text { Variable }\end{array}$} \\
\hline & PMS & EUA & $\mathrm{NH}$ & $\mathrm{H} / \mathrm{F}$ & DIC & $\mathrm{N}$ & NT & NDA & NTDA \\
\hline & $\begin{array}{c}D M Y \\
\left({\left.\mathrm{Mg} \cdot \mathrm{ha}^{-1}\right)}^{2}\right.\end{array}$ & $\begin{array}{c}W U E \\
\left(\mathrm{~kg} \cdot \mathrm{mm}^{-1}\right)\end{array}$ & $\begin{array}{c}N S \\
\left(\mathrm{~m}^{2}\right)\end{array}$ & $S / L$ & $\begin{array}{c}C I D \\
\left(\Delta^{13} \mathrm{CPDB}\right)\end{array}$ & $\begin{array}{c}N \\
(\%)\end{array}$ & $\begin{array}{c}T N \\
\left(\mathrm{~kg}^{\left.-h a^{-1}\right)}\right.\end{array}$ & $\begin{array}{l}N D A \\
(\%)\end{array}$ & $\begin{array}{c}\text { TNDA } \\
\left(\mathrm{kg}^{\left.-\mathrm{ha}^{-1}\right)}\right.\end{array}$ \\
\hline$\overline{\mathrm{PMS}(D M Y)}$ & - & $0,98 * *$ & 0,33 & 0,19 & $-0,15$ & $-0,56 *$ & $0,94 * *$ & 0,20 & 0,43 \\
\hline EUA (WUE) & $0,98 * *$ & - & 0,35 & 0,28 & $-0,19$ & $-0,55^{*}$ & $0,93 * *$ & 0,08 & 0,31 \\
\hline NH (NS) & $-0,40$ & $-0,34$ & - & $-0,26$ & 0,13 & 0,30 & 0,51 & $-0,14$ & 0,23 \\
\hline $\mathrm{H} / \mathrm{F}(\mathrm{S} / L)$ & 0,50 & $0,58 *$ & $-0,30$ & - & $-0,59 *$ & $-0,05$ & 0,20 & $-0,33$ & $-0,26$ \\
\hline $\mathrm{DIC}(C I D)$ & $0,51^{*}$ & 0,47 & $-0,24$ & $-0,17$ & - & $-0,08$ & $-0,19$ & 0,02 & 0,24 \\
\hline $\mathrm{N}(N)$ & $-0,68 * *$ & $-0,69 *$ & $0,62 *$ & $-0,74 * *$ & $-0,05$ & - & $-0,24$ & $-0,38$ & 0,02 \\
\hline NT (TN) & $0,92 * *$ & $0,89 * *$ & $-0,22$ & 0,23 & $0,62 *$ & $-0,35$ & - & 0,06 & 0,51 \\
\hline NDA (NDA) & 0,09 & 0,16 & 0,09 & $-0,07$ & 0,10 & 0,09 & 0,15 & - & $0,56 *$ \\
\hline NTDA (NTDA) & $0,74 * *$ & $0,71 * *$ & $-0,14$ & 0,03 & $0,51^{*}$ & $-0,31$ & $0,78 * *$ & 0,38 & - \\
\hline
\end{tabular}

${ }^{1}$ PMS (DMY) produção de matéria seca (dry matter yield), EUA (WUE) eficiência do uso da água (water use efficiency), NH (NS) número de hastes (number of steams), H/F (S/L) relação haste/folha (steam/leave ratio), DIC (CID) discriminação isotópica de carbono (carbo isotopic discrimination), N (N) teor de nitrogênio (average of nitrogen), NT (TN) nitrogênio total (total nitrogen), NDA (NDA) nitrogênio derivado da atmosfera (nitrogen derived from atmosphere) e NTDA (TNDA) nitrogênio total derivado da atmosfera (total nitrogen derived from atmosphere) $*(P<0,05){ }^{* *}(P<0,01)$. 
Tabela 5 - Desempenho de cultivares de alfafa mantidos em lisímetros sob condições de irrigação controlada e regime de chuvas. Média de 4 repetições e 10 cortes no experimento 2

Table 5 - Performance of alfalfa cultivars maintained in lisymeters under irrigated and rain-fed conditions. Average of 4 replications and 10 cuts experiment 2

\begin{tabular}{|c|c|c|c|c|c|c|c|c|c|}
\hline \multirow{3}{*}{$\begin{array}{l}\text { Cultivares } \\
\text { Cultivars }\end{array}$} & \multicolumn{9}{|c|}{$\begin{array}{l}\text { Variável } \\
\text { Variable }\end{array}$} \\
\hline & PMS & EUA & $\mathrm{NH}$ & $\mathrm{H} / \mathrm{F}$ & DIC & $\mathrm{N}$ & NT & NDA & NTDA \\
\hline & $\begin{array}{c}D M Y \\
\left(\mathrm{Mg}^{\left.-\mathrm{ha}^{-1}\right)}\right.\end{array}$ & $\begin{array}{c}\text { WUE } \\
\left(\mathrm{kg} \cdot \mathrm{mm}^{-1}\right)\end{array}$ & $\begin{array}{c}N S \\
\left(\mathrm{~m}^{2}\right)\end{array}$ & $S / L$ & $\begin{array}{c}C I D \\
\left(\Delta^{13} \mathrm{CPDB}\right)\end{array}$ & $\begin{array}{c}N \\
(\%)\end{array}$ & $\begin{array}{c}T N \\
\left(\mathrm{~kg} \cdot \mathrm{ha}^{-1}\right)\end{array}$ & $\begin{array}{c}N D A \\
(\%)\end{array}$ & 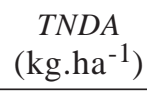 \\
\hline \multicolumn{10}{|l|}{ Irrigação (Irrigated) } \\
\hline Crioula Brasil & 2,54 & 2,67 & 721 & 0,88 & 20,92 & 3,33 & 82,05 & 82,22 & 65,88 \\
\hline Crioula Chile & 2,29 & 2,52 & 645 & 0,90 & 21,08 & 3,16 & 71,63 & 81,11 & 57,92 \\
\hline UC-Cíbola & 2,37 & 2,57 & 694 & 0,95 & 21,02 & 3,14 & 73,06 & 81,50 & 59,39 \\
\hline WL-516 & 2,20 & 2,42 & 668 & 0,98 & 21,08 & 3,12 & 68,36 & 81,44 & 55,77 \\
\hline Месса & 2,19 & 2,35 & 670 & 0,80 & 21,19 & 3,21 & 69,03 & 82,73 & 56,79 \\
\hline PioneerXAI32 & 2,36 & 2,57 & 701 & 0,81 & 21,05 & 3,30 & 76,33 & 84,28 & 63,85 \\
\hline DMS(0.05) & 0,67 & 0,70 & 95 & 0,12 & 0,26 & 0,15 & 19,32 & 1,88 & 13,55 \\
\hline $\mathrm{CV}$ & 12,56 & 12,07 & 5,56 & 5,75 & 0,54 & 2,12 & 11,49 & 0,99 & 9,84 \\
\hline Pr. $>$ F & 0,5945 & 0,7136 & 0,1119 & $0,0006^{* *}$ & 0,0378* & $0,0018 * *$ & 0,2555 & $0,0007^{* *}$ & * 0,1554 \\
\hline \multicolumn{10}{|l|}{ Chuva (Rainfall) } \\
\hline Crioula Brasil & 1,33 & 1,46 & 587 & 0,63 & 20,53 & 3,56 & 46,03 & 63,60 & 32,47 \\
\hline Crioula Chile & 1,33 & 1,48 & 552 & 0,67 & 20,49 & 3,44 & 44,87 & 58,00 & 28,38 \\
\hline UC-Cíbola & 1,22 & 1,35 & 550 & 0,62 & 20,57 & 3,43 & 40,94 & 58,80 & 26,36 \\
\hline WL-516 & 1,11 & 1,23 & 586 & 0,61 & 20,42 & 3,55 & 38,90 & 70,97 & 29,33 \\
\hline Месса & 1,01 & 1,15 & 504 & 0,55 & 20,87 & 3,46 & 34,27 & 66,17 & 24,29 \\
\hline PioneerXAI32 & 0,91 & 1,04 & 495 & 0,63 & 20,45 & 3,48 & 31,39 & 66,23 & 23,09 \\
\hline $\operatorname{DMS}(0.05)$ & 0,28 & 0,28 & 92 & 0,05 & 0,19 & 0,29 & 8,48 & 6,88 & 5,87 \\
\hline $\mathrm{CV}$ & 10,78 & 9,48 & 7,54 & 3,51 & 0,41 & 3,67 & 9,36 & 4,67 & 9,36 \\
\hline Pr. $>$ F & $0,0008^{* *}$ & $0,0006^{* *}$ & $0,0246^{*}$ & $0,0001^{* *}$ & $0,0001^{* *}$ & 0,6298 & $0,0003^{* *}$ & $0,0002^{* *}$ & * $0,0012^{* *}$ \\
\hline
\end{tabular}

ções de irrigação a PMS correlacionou-se positivamente com a EUA $\left(0,96^{* *}\right), \mathrm{NH}\left(0,80^{*}\right)$, NT $\left(0,97^{* *}\right)$ e NTDA $\left(0,93^{* *}\right)$ e, negativamente com a DIC ($0,92 * *)$. Sob condições de chuva, a PMS correlacionou-se à EUA $(0,99 * *)$, NT $(0,99 * *)$ e NTDA $\left(0,81^{*}\right)$. Em relação à EUA, no experimento sob irrigação, observa-se correlação negativa com a DIC $\left(-0,94^{* *}\right)$, conforme descrito na Tabela 6 .

Nesse experimento, a evapotranspiração variou entre 1,7 a 7,1 mm.dia ${ }^{-1}$ no início e momento do corte, respectivamente. A produção da alfafa relaciona-se diretamente a evapotranspiração da cultura e a água aplicada (Hanson \& Orloff, 1998), sendo a eficiência do uso da água a relação entre a água aplicada e a quantidade utilizada ou, ainda, a água necessária aplicada em relação à água utilizada, uma vez que a evapotranspiração da cultura é crescente, conforme descrito por Rassini \& Leme (2001) em trabalho com o cultivar Crioula. A determinação da evapotranspiração pelo $\mathrm{K}_{\mathrm{c}}$ e $\mathrm{E}_{\text {to }}$ estimada em tanque classe A (Doorenbos \& Kassan, 1979) tem sido utilizada com sucesso para a cultura da alfafa, conforme descrito por Metochis (1980) e Undersander (1986). Os resultados evidenciaram estreita e positiva relação entre a PMS e EUA independente do ambiente de estudo, resultando em maior produção e eficiência de transpiração. A base fisiológica para variação da DIC em plantas $C_{3}$ pode ser resumida em termos da razão da concentração do $\mathrm{CO}_{2}$ interno e externo, com tendência de correlação positiva entre a PMS e DIC. Entretanto, essa correlação pode ser negativa em ambientes em que a água seja limitante (Faruquhar \& Richards, 1984) ou quando o conjunto de cultivares analisados se mostrem contrastantes (Ehleringer et al., 1991). Segundo Condon et al. (1987) se a correlação positiva entre a DIC e PMS for uma regra, não existe possibilidade de aumento da EUA, uma vez que o aumento da DIC é resultado do aumento da transpiração. Nos experimentos a PMS dos cultivares mantidos sob irrigação correlacionaram- 
se negativamente com a DIC, resultados semelhantes ao de Johnson \& Tieszen (1994) em estudo com 18 cultivares de alfafa. Uma hipótese para justificar os resultados pode estar relacionada aos cultivares estudados que variam quanto à eficiência na utilização da água absorvida no processo de fotossíntese, diminuindo a necessidade de manutenção dos estômatos abertos e resultando em correlação positiva entre a EUA e PMS. Corrobora com essa hipótese a alta concentração de nitrogênio, indispensável à composição das enzimas do sistema fotossintético como a RuBisCO. Outra hipótese seria a análise em separado dos dados obtidos nos experimentos sob irrigação e chuva cuja análise conjunta resultou em correlação positiva invariavelmente (Matus et al., 1995). Nesse experimento, a análise de correlação linear entre PMS e DIC utilizando todo o conjunto de dados resultou em $r=0,70$, quando avaliado o conjunto de 12 cultivares. Entretanto, quando avaliados os dois conjuntos em separado, observa-se correlação negativa entre a PMS e DIC de $r=-0,92 * *$, para plantas mantidas sob irrigação, e $\mathrm{r}=-0,23$ para plantas sob regime de chuvas (Figura 1 ).

A seleção de plantas para aumento da produtividade com base no aumento da DIC, conforme White et al. (1990), não levou em consideração outras limitações como a FBN e teor de nitrogênio que reflete a concentração de enzimas como a RuBisCO e, segundo o autor essa relação foi inconsistente para plantas de feijoeiro mantidas em diferentes locais.

Para plantas $\mathrm{C}_{3}$, a DIC correlaciona-se negativamente com a EUA (Farquhar et al., 1982) e fatores como sistema radicular e adaptação às condições climáticas são fundamentais (Hubich et al., 1986; White et al., 1990; Johnson \& Tieszen, 1994). Nesse estudo, essa correlação foi observada somente quando se restringiu o número de cultivares e estendeu-se o período de avaliação para 12 meses. Para programas de seleção de cultivares Ray et al. (1998) propuseram a identificação de cultivares com maior desenvolvimento radicular, o que facilitaria, sobremaneira, a adaptação ao déficit hídrico, por exemplo. Sob déficit hídrico a relação haste/folha diminui e o teor de proteína bruta aumenta (Oliveira, 1986) aumentando a qualidade e resultando em correlação negativa com a produção (Denment et al., 1986; Robinson et al., 1994). Sob irrigação, o desenvolvimento dos cultivares mais produtivas é otimizado, com menor estacionalidade(Botrel \& Alvim, 1997; Rassini \& Leme, 2001), devido ao fornecimento de água na época de seca. Essa variação é menor para cultivares que possuem maior capacidade de ajustamento osmótico e manutenção do turgor, mesmo sob baixo potencial hídrico (Wright et al., 1996), como é o caso dos cultivares Crioulas, que possuem ampla adaptação.

O nitrogênio utilizado pela alfafa é uma combinação entre o $\mathrm{N}$ absorvido do solo e o NDA obtido via FBN, sendo a alfafa uma planta hábil na utilização

Tabela 6 - Correlações lineares entre características de produção e qualidade da alfafa mantidas sob regime de irrigação (acima da diagonal) e regime de chuva (abaixo da diagonal). Média de 4 repetições e 10 cortes no experimento 2

Table 6 - Linear correlations among traits associated with yield and quality of the alfalfa maintained under irrigated (above diagonal) and rainfed conditions (below diagonal). Average of 4 replications and 10 cuts in the experiment 2

\begin{tabular}{|c|c|c|c|c|c|c|c|c|c|}
\hline \multirow{3}{*}{$\begin{array}{l}\text { Cultivares } \\
\text { Cultivars }\end{array}$} & \multicolumn{9}{|c|}{$\begin{array}{l}\text { Variável } \\
\text { Variable }\end{array}$} \\
\hline & PMS & EUA & $\mathrm{NH}$ & $\mathrm{H} / \mathrm{F}$ & DIC & $\mathrm{N}$ & NT & NDA & NTDA \\
\hline & $\begin{array}{c}D M Y \\
\left(\mathrm{Mg}^{\mathrm{hg}} \mathrm{ha}^{-1}\right)\end{array}$ & $\begin{array}{c}\text { WUE } \\
\left(\mathrm{kg} \cdot \mathrm{mm}^{-1}\right)\end{array}$ & $\begin{array}{c}N S \\
\left(\mathrm{~m}^{2}\right)\end{array}$ & $S / L$ & $\begin{array}{c}C I D \\
\left(\Delta^{13} \text { CPDB }\right)\end{array}$ & $\begin{array}{c}N \\
(\%)\end{array}$ & $\begin{array}{c}T N \\
\left(\mathrm{~kg}^{\mathrm{h}} \mathrm{ha}^{-1}\right)\end{array}$ & $\begin{array}{c}N D A \\
(\%)\end{array}$ & 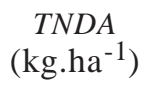 \\
\hline PMS (DMY) & - & $0,96 * *$ & $0,80 *$ & - & 0,37 & 0,36 & 0,64 & $0,87 *$ & $-0,06$ \\
\hline EUA (WUE) & $0,99 * *$ & - & 0,69 & 0,03 & $-0,92 * *$ & 0,68 & $0,97 * *$ & 0,14 & $0,93 * *$ \\
\hline $\mathrm{NH}(N S)$ & 0,75 & 0,71 & - & 0,08 & $-0,94 * *$ & 0,56 & $0,91^{* *}$ & 0,08 & $0,87^{*}$ \\
\hline $\mathrm{H} / \mathrm{F}(S / L)$ & 0,55 & 0,55 & 0,35 & $-0,17$ & $-0,72 *$ & 0,73 & $0,82 *$ & 0,48 & $0,85^{*}$ \\
\hline $\mathrm{DIC}(C I D)$ & $-0,23$ & $-0,21$ & $-0,45$ & - & $-0,31$ & $-0,67$ & $-0,22$ & $-0,79 *$ & $-0,32$ \\
\hline $\mathrm{N}(N)$ & 0,07 & 0,04 & 0,59 & $-0,82^{*}$ & - & $-0,46$ & $-0,85^{*}$ & 0,07 & $-0,79 *$ \\
\hline $\mathrm{NT}(T N)$ & $0,99 * *$ & $0,99 * *$ & $0,81^{*}$ & $-0,04$ & $-0,31$ & - & $0,83 *$ & 0,70 & $0,89 *$ \\
\hline NDA (NDA) & $-0,62$ & $-0,64$ & 0,01 & 0,55 & $-0,28$ & 0,19 & - & 0,33 & $0,98 * *$ \\
\hline NTDA $(N T D A)$ & $0,81^{*}$ & $0,79 *$ & $0,93 * *$ & $-0,52$ & 0,01 & 0,66 & $-0,53$ & - & 0,48 \\
\hline
\end{tabular}

${ }^{1}$ PMS (DMY) produção de matéria seca (dry matter yield), EUA (WUE) eficiência do uso da água (water use efficiency), NH (NS) número de hastes (number of steams), H/F (S/L) relação haste/folha (steam/leave ratio), DIC (CID) discriminação isotópica de carbono (carbo isotopic discrimination), $\mathrm{N}(\mathrm{N})$ teor de nitrogênio (average of nitrogen), NT (TN) nitrogênio total (total nitrogen), NDA (NDA) nitrogênio derivado da atmosfera (nitrogen derived from atmosphere) e NTDA (TNDA) nitrogênio total derivado da atmosfera (total nitrogen derived from atmosphere). $*(P<0,05) * *(P<0,01)$. 
tanto da combinação quanto de uma das duas fontes isoladas (Teuber \& Phillips, 1986). A diferenciação entre as duas fontes pode ser obtida pela análise do fracionamento isotópico (Shearer \& Kohl, 1997). A proporção do N obtido via FBN declina rapidamente quando existe $\mathrm{N}$ na solução do solo, mas tende a estabilizar após o segundo corte, devido a alta capacidade de extração de nitrogênio do solo pela alfafa (Hogh-Jensen \& Schjoerring, 1997). Durante 3 anos consecutivos de experimentação com alfafa nos pampas da Argentina, Basigalup (2000), encontraram índices de $42 \%$ para NDA e $200 \mathrm{~kg} \cdot \mathrm{ha}^{-1}$. ano ${ }^{-1}$ para NTDA. Para a alfafa, 50 a $80 \%$ do nitrogênio nas folhas está alocado na forma de proteína fotossintética (Evans, 1989). Parte do nitrogênio (40 a 60\%) é transferido para raízes e coroas da alfafa e mobilizado para a emissão e desenvolvimento da parte aérea após o corte. A combinação entre $\mathrm{N}$ de reserva e $\mathrm{N}$ obtido via fixação tende a variar dentro de um ciclo de produção decrescendo a parcela de reserva para 87, 66 e 52\%, após 3, 10 e 30 dias após o corte, respectivamente.

Em linhas gerais, a FBN é positivamente correlacionada a PMS (Heichel et al., 1984) e DIC (Knight et al., 1993), uma vez que existe uma interdependência entre as partes. O metabolismo do $\mathrm{N}$ influencia a DIC por estar envolvido na formação da enzima RuBisCO responsável pela assimilação do carbono e principal passo fisiológico na discrimina-

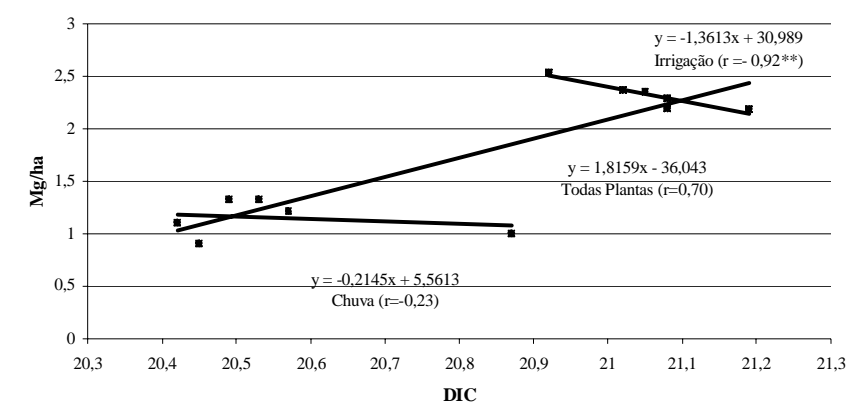

Figura 1 - Correlação entre médias de produção de matéria seca $\left(\mathrm{Mg} \mathrm{ha}^{-1}\right)$ e discriminação isotópica do carbono (DIC) de forma conjunta e separadamente para dados obtidos sob irrigação e chuva no experimento 2 .

Figure 1 - Correlations between dry matter yield (Mg.ha-1) and carbon isotopic discrimination (CID) of date obtained under irrigation and rain-fed conditions in the experiment 2. ção dos isótopos de carbono em plantas $C_{3}$. Sob condições de estresse hídrico ou salino o processo pode ser alterado resultando até em relação negativa como obtido por Kumarasinghe et al. (1992) em soja. No experimento 1, os dados são inconsistentes e não descrevem uma tendência, possivelmente por não representarem um ciclo anual. No experimento 2 a condição de estresse hídrico promoveu um desequilíbrio entre as relações, que puderam ser melhor visualizadas na condição de irrigação, em que a correlação positiva entre DIC e FBN foi evidenciada, confirmando a tendência descrita por Knight et al. (1993) para leguminosas eficientes quanto à FBN. Sob condições de irrigação, para uma produção anual de 24,5 Mg.ha-1, a simbiose alfafa/Sinorhizobium meliloti introduziu até $116,69 \%$ a mais de nitrogênio a cada corte em relação ao regime de chuvas.

\section{Conclusões}

Sob condições controladas a alfafa responde positivamente à irrigação, tanto para a produção de matéria seca quanto para obtenção de nitrogênio e dentre os cultivares estudados, os cultivares Crioula Brasileira e Crioula Chilena apresentam os melhores desempenhos.

A correlação significativa e positiva entre produção de matéria seca e eficiência do uso da água foi observada tanto sob regime de irrigação quanto regime de chuvas, entretanto a correlação entre eficiência do uso da água e discriminação isotópica do carbono foi inconsistente em regime de chuvas e significativamente negativa sob irrigação.

O potencial de fixação biológica do nitrogênio (NDA) correlaciona-se positivamente com a produção de matéria seca para o ciclo de um ano.

\section{Agradecimento}

À Fundação de Amparo à Pesquisa do Estado de São Paulo (FAPESP), pelo suporte financeiro.

\section{Literatura Citada}

ARAÚJO, R.S.; HUNGRIA, M. Microrganismos de importância agrícola. Brasília: EMBRAPA, CNPAF, CNPSo, 1994. 236p.

ASSENG, S.; HSIAO, T.C. Canopy $\mathrm{CO}_{2}$ assimilation, energy balance and water use efficiency of an alfalfa crop before and after cutting. Field Crop Research, v.67, p.191-206, 2000. 
ATKINS, C.A. Efficiencies and inefficiencies in the legume/ Rhizobium symbiosis - a review. Plant and Soil, v.82, p.273-284, 1984.

BASIGALUP, D.H. Alfalfa dinitrogen fixation in Manfredi, Argentina. In: NORTH AMERICAN ALFALFA IMPROVEMENT CONFERENCE, 37., 2000, Madison. Proceedings... Madison: 2000. p.325.

BARRIE, A.; PROSSER, S.J. Automated analysis of lightelement stable isotope by ratio mass spectrometry. In: BOUTTON, T.W.; YAMASAKI, S. (Eds.) Mass spectrometry of soils. New York: Marcel Dekker, 1997. p.1-46.

BOTREL, M.A.; ALVIM, M.J. Avaliação de cultivares de alfafa na zona da mata de Minas Gerais. Pesquisa Agropecuária Brasileira, v.32, n.9, p.971-975, 1997.

BOUTTON, T.W. Stable carbon isotope ratios of soil organic matter and their use a indicators of vegetation and climate change. In: BOUTTON, T.W., YAMASAKI, S. (Eds.) Mass spectrometry of soils. New York: Marcel Dekker, 1997. p.47-82.

CONDON, A.G.; RICHARDS, R.A.; FARQUHAR, G.D. Carbon isotope discrimination is positively correlation with grain yield and dry matter production in field-grown wheat. Crop Science, v.27, p.997-1001, 1987.

CUNHA, G.R., PAULA, J.R.F., BERGAMASCHI, H. et al. Evapotranspiração e eficiência no uso da água em alfafa. Revista Brasileira de Agrometereologia, v.2, p.23-27, 1994.

DENMENT, M.W., TEUBER, L.R., BOURQUE, D.P. et al. Changes in forage quality of improved alfalfa populations. Crop Science, v.26, p.1137-1146, 1986.

DOORENBOS, J.; KASSAM, A.M. Efectos del agua sobre el rendimiento de los cultivos. Roma: FAO, 1979. 212p.

EBSON, J.S.; PETROVIC, A.M.; DAWSON, T.E. Relationship between carbon isotope discrimination, water use efficiency and evapotranspiration in Kentucky bluegrass. Crop Science, v.38, p.157-162, 1998.

EHLERINGER, J.; KLASSEN, S.; CLAYTON, C. et al. Carbon isotope discrimination and transpiration efficiency in common bean. Crop Science, v.31, p.1611-1615, 1991.

EVANS, J.R. Photosynthesis and nitrogen relationships in leaves of $\mathrm{C}_{3}$ plants. Oecologie, v.78, p.9-19, 1989.

FARQUHAR, G.D.; O'LEARY, M.H.; BERRY, J.A. On the relationship between carbon isotope discrimination and the intercellular carbon dioxide concentration in leaves. Australian Journal of Plant Physiology, v.9, p.121-137, 1982.

FARQUHAR, G.D.; RICHARDS, R.A. Isotopic composition of plant carbon correlates with water-use efficiency of wheat genotypes. Field Crops, v.11, p.539-552, 1984.

FARQUHAR, G.D.; LLOYD, J. Carbon and oxygen isotope effects in the exchange of carbon dioxide between terrestrial plants and atmosphere. In: EHLERINGER, J.R.; HALL, A.E.; FARQUHAR, G.D. (Eds.) Stable isotope and plant carbon-water relations. San Diego: Academic Press, 1993. p.47-70.

FISHBECK, K.A.; HEICHEL, G.H.; VANCE, C.P. Dry matter, nitrogen distribution and nitrogen fixation in contrasting alfalfa symbioses. Crop Science, v.27, p.1205-1209,1987.

GOMES, F.P. A estatística moderna na agropecuária. Piracicaba: Potafós, 1987. 160p.

HALL, A.E.; MUTTER, R.G.; HUBICK, K.T. et al. Genotypic differences in carbon isotope discrimination in cowpea under wet and dry field conditions. Crop Science, v.30, p.300-
305, 1990.

HANSON, B.;ORLOFF, S. Double your yield with half the water: Is it possible? In: 1998 CALIFORNIA/NEVADA ALFALFA SYMPOSIUM, 1998, Davis. Proceedings ... Davis: University of California, 1998. p.75-87.

HARDARSON, G.; ZAPATA, F.; DANSO, S.K.A. Field evaluation of symbiotic nitrogen fixation by rhizobial strain using ${ }^{15} \mathrm{~N}$ methodology. Plant and Soil, v.82, p.369-375, 1984.

HEICHEL, G.H., HARDARSON, G., BARNES, D.K. et al. Dinitrogen fixation, herbage yield, and rhizobial preference of selected alfalfa clones. Crop Science, v.24, p.1093-1097, 1984.

HOGH-JENSEN, H.; SCHJOERRING, J.K. Effects of drought and inorganic $\mathrm{N}$ form on nitrogen fixation and carbon isotope discrimination in Trifolium repens. Plant Physiology Biochemistry, v.35, p.55-62, 1997.

HUBICK, K.T.; FARQUHAR, G.D.; SHORTER, R. Correlation between water-use efficiency and carbon isotope discrimination in diverse peanut (Arachis) germplasm. Australian Journal of Plant Physiology, v.13, p.803-816, 1986.

JASSEN, D.L.; BARNES, D.K.; VANCE, C.P. et al. Variation for activity of nodule nitrogen and carbon assimilating enzymes in alfalfa. Crop Science, v.27, p.627-631, 1994.

JOHNSON, R.C.; TIESZEN, L.L. Variation for water use efficiency in alfalfa germplasm. Crop Science, v.34, p.452-458, 1994.

KNIGHT, J.D.; VERHEES, F.; Van KESSEL, C. et al. Does carbon isotope discrimination correlate with biological nitrogen fixation? Plant Soil, v.153, p.151-153, 1993.

KUMARASINGHE, K.S.; KIRDA, C.; MOHAMED, A. et al. ${ }^{13} \mathrm{C}$ isotope discrimination correlates with biological nitrogen fixation (Glycine max L. merrill). Plant Soil, v.139, p.145-147, 1992.

MARIOTTI, A.; MARIOTTI, F.; AMARGER, N. et al. Fractionnements isotopiques de lázote lors des processus d'absorption des nitrates et de fixation del, azote atmposphérique par les plantes. Physiologie Végetale, v.8, p.163-181, 1980.

MATUS, A.; SLINKARD, A.E.; Van KESSEL, C. Carbon isotope discrimination and indirect selection for seed yield in lentil. Crop Science, v.35, p.679-684, 1995.

METOCHIS, C. Irrigation of lucerne under semi-arid conditions in Cyprus. Irrigation Science, v.1, p.247-252, 1980.

OLIVEIRA, P.R. Avaliação da produção de feno de alfafa (Medicago sativa L.). Piracicaba: Escola Superior de Agricultura "Luiz de Queiroz”, 1986. 75p. Dissertação (Mestrado) - Escola Superior de Agricultura "Luiz de Queiroz”, 1986.

OLIVEIRA, W.S.; OLIVEIRA, P.P.A.; TSAI, S.M. Associações simbióticas com a microbiota do solo. In: SIMPÓSIO SOBRE MANEJO DE PASTAGENS, 16., 1999, Piracicaba. Anais ... Piracicaba: Fundação de Estudos Agrários “Luiz de Queiroz”, 1999. p.117-132.

OLIVEIRA, W.S. Seleção de cultivares de alfafa (Medicago sativa $L$.) eficientes em produção e qualidade da biomassa. Piracicaba: Universidade de São Paulo, 2001. 118p. Tese (Doutorado) - Universidade de São Paulo, 2001.

RACCA, R.; BASIGALUP, D.; BRENZONI, E. et al. Alfalfa symbiotic dinitrogen fixation in the Argentine Pampean Region. In: NORTH AMERICAN ALFALFA IMPROVEMENT CONFERENCE, 36., 1998, Bozeman. 
Proceedings ... Bozeman, Montana, 1998. p.71.

RAY, I.M.; TOWNSEND, M.S.; MUNCY, C.M. Variation for yield, water use efficiency and canopy morphology among nine alfalfa germplasm. Crop Science, v.38, p.1386-1390, 1998.

RASSINI, J.B.; LEME, E.J.A. Water management for establishment of alfalfa (Medicago sativa). In: INTERNATIONAL GRASSLAND CONFERENCE, 19., 2001, Piracicaba. Proceedings ... Piracicaba: Fundação de Estudos Agrários “Luiz de Queiroz”, 2001. p.591.

ROBINSON, F.E.; TEUBER, L.R.; GIBBS, L.K. Alfalfa water stress management during summer in Imperial Valley for water conservation. El Centro, CA: Desert Research and Extension Center, 1994. 57p.

SHEARER, G.; KOHL, D.H. Natural abundance of ${ }^{15} \mathrm{~N}$ : Fractional contribution of two sources to a common sink and use of isotope discrimination. In: BOUTTON, T.W., YAMASAKI, S. (Eds.) Mass spectrometry of soils. New York: Marcel Dekker, 1997. p.89-125.

SOMASEGARAN, P.; HOBEN, H.J. Handbook for Rhizobia. Methods in legume-Rhizobia technology. New York: Springer-Verlag, 1994. 145p.

TEUBER, L.R.; PHILLIPS, D.A. Influences of selection method and nitrogen environment on breeding alfalfa for increased forage yield and quality. Crop Science, v.28, p.599-604, 1986.
UNDERSANDER, D.J. Alfalfa (Medicago sativa L.) growth response to water and temperature. Irrigation Science, v.8, p.23-33, 1986.

WHite, J.W.; CASTILLO, J.A.; EHLERINGER, J. Associations between productivity, root growth and carbon isotope discrimination in Phaseolus vulgaris under water deficit. Australian Journal of Plant Physiology, v.17, p.189-198, 1990.

WRIGHT, G.C.; WHITFIELD, D.M.; GYLES, O.A. et al. Effects of frequency of irrigation and gypsum treatment on leaf water potential and leaf stomatal conductance of lucerne (Medicago sativa L.) grown on a clay soil. Irrigation Science, v.7, p.73-82, 1996.

ZHU, Y.P.; SHEAFFER, C.C.; RUSSELLE, M.P. et al. Dinitrogen fixation of annual Medicago species. In: NORTH AMERICAN ALFALFA IMPROVEMENT CONFERENCE, 35., 1996, Oklahoma City. Proceedings ... Oklahoma City, 1996. p.75.
Recebido em: 05/06/02

Aceito em: 10/03/03 